Linguistique, littérature, didactique

\title{
Concepts et modèles en didactique du français : introduction
}

\section{Nathalie Denizot et Claudine Garcia-Debanc}

\section{(2) OpenEdition \\ 1 Journals}

\section{Édition électronique}

URL : https://journals.openedition.org/pratiques/9434

DOI : 10.4000/pratiques.9434

ISSN : 2425-2042

Éditeur

Centre de recherche sur les médiations (CREM)

\section{Référence électronique}

Nathalie Denizot et Claudine Garcia-Debanc, «Concepts et modèles en didactique du français : introduction », Pratiques [En ligne], 189-190 | 2021, mis en ligne le 09 juillet 2020, consulté le 23 juillet 2021. URL : http://journals.openedition.org/pratiques/9434 ; DOI : https://doi.org/10.4000/pratiques. 9434

Ce document a été généré automatiquement le 23 juillet 2021.

(c) Tous droits réservés 


\title{
Concepts et modèles en didactique du français : introduction
}

\author{
Nathalie Denizot et Claudine Garcia-Debanc
}

Dans le numéro 149-150 de Pratiques intitulé Didactique du français 2, B. Daunay et Y. Reuter (2011) portaient un regard critique sur les recherches en didactique du français et mettaient en évidence les fragilités de la didactique du français comme discipline de recherche d'un triple point de vue :

(a) les problèmes institutionnels liés aux politiques éducatives, aux modes d'évaluation des recherches et à l'instabilité de la formation des maitres ;

(b) la grande «sensibilité sociale » de la didactique du français et son inscription dans des cursus disciplinaires divers ;

(c) les problèmes scientifiques, avec la difficulté à s'émanciper des «disciplinesmères » et les débats avec les disciplines contributoires.

Ils appelaient de leurs vœux « une clarification conceptuelle et méthodologique interne " (ibid., p. 20) et considéraient comme indice de maturité scientifique de pouvoir «fonder son cadre théorique sur des concepts spécifiques éprouvés collectivement ». C'était là précisément le projet du colloque 2019 de l'AIRDF à Lyon, dont le présent numéro rassemble certaines contributions.

Ainsi cette publication prend la suite de plusieurs numéros thématiques de Pratiques consacrés à la Didactique du Français: 137-138, La didactique du français. Hommages à Jean-François Halté (Garcia-Debanc \& Masseron, 2008) ; 145-146, Didactique du français 1 (Halté \& Petitjean, 2010); et 149-150, Didactique du français 2 (Halté \& Petitjean, 2011). Notre objectif dans le présent numéro est de faire un point épistémologique, une dizaine d'années après ces publications, sur les concepts en usage dans le champ de la didactique $d u$ français langue première au fur et à mesure de sa maturation comme discipline de recherche.

Le numéro est alimenté par des contributions - remaniées pour cette publication issues du colloque d'aout 2019 de l'AIRDF (Association Internationale pour la Recherche en Didactique du Français ${ }^{1}$ ), qui portait sur cette problématique et qui avait comme titre : « Les concepts dans la didactique du français. Émergence et création d'un champ épistémique ». C'est ainsi l'occasion de faire un état de lieux du champ de la didactique $\mathrm{du}$ français comme discipline scientifique à un temps T, en l'occurrence en 2019-2020. 
Nous avons veillé à ce que soient représentés dans ce numéro, les différents pays de la francophonie (conformément aux missions de l'AIRDF) et les différentes composantes de la discipline «français » dans leur diversité : lecture-écriture et étude de la langue (lexique, orthographe, grammaire), littérature... Toutefois, il faut rester prudent et considérer que cet état des lieux est partiel, à la fois parce qu'il ne peut rendre compte que des contributions que les participants au colloque ont proposées, mais aussi parce que la publication des contributions de ce colloque a été répartie en trois supports: outre le présent numéro, il faut ajouter un ouvrage à paraitre aux Presses Universitaires du Septentrion ainsi que le dossier du numéro 67 de la revue La Lettre de l'AIRDF.

\section{Un point d'étape dans l'histoire d'une discipline de recherche récente : la didactique du français}

\section{La didactique du français langue première en $\mathbf{2 0 2 0}$}

4 La comparaison de la situation décrite dans les numéros précédents de Pratiques en2010 et 2011 avec celle de la conjoncture actuelle, fait apparaitre un certain nombre de faits marquants que nous analyserons en reprenant les distinctions posées par É. Nonnon (2010) selon les dimensions suivantes:

- l'enseignement du français comme discipline scolaire, s'inscrivant dans une longue tradition,

- la didactique du français comme discipline de recherche, construisant progressivement son autonomie conceptuelle et méthodologique,

- la didactique du français comme discipline de formation, les recherches en didactique du français alimentant les activités de formation initiale et continue, ce qui correspond aux enjeux praxéologiques de la didactique.

Du point de vue de la discipline scolaire français, la situation en France se caractérise par des changements profonds de programmes scolaires, au gré des alternances politiques (2015, avec des ajustements en 2018 et 2020) et des réformes majeures, notamment du baccalauréat. Dans les autres pays de la francophonie (Belgique, Québec, Suisse), la stabilité dans cette période est plus grande, comme le montre l'analyse comparative des programmes dans l'ouvrage collectif consacré à la Didactique du français langue première (Simard et al., 2019, p. 115-140)

Du point de vue de la didactique du français comme discipline scientifique, la décennie se caractérise par une forme d'autonomisation de réseaux de chercheurs, notamment en didactique de la littérature (Daunay, 2007 ; Ahr, 2015 ; Denizot, Dufays \& Louichon, 2019), par la soutenance de nombreuses thèses dans les différents pays de la francophonie et surtout par la mise en place de grandes recherches - dont certaines sont d'ailleurs internationales - portant sur des effectifs importants en nombre de classes et d'élèves observés, pour décrire les pratiques d'enseignement et les mettre en regard de leurs effets sur les apprentissages. Ainsi, en France, la recherche Lire et écrire CP menée par R. Goigoux sur les «pratiques efficaces » d'apprentissage du lire-écrire a mobilisé entre 2013 et 2015: 60 chercheurs issus de 13 universités et plus de 70 collaborateurs, et a porté sur 2507 élèves issus de 131 classes observées dans 16 académies $^{2}$. En Suisse, l'équipe du Grafe (Groupe de recherche pour l'analyse du français enseigné) a mené ces dernières années, plusieurs recherches dans plusieurs 
cantons suisses, notamment autour de la lecture et de l'enseignement des textes littéraires ${ }^{3}$, en analysant des séquences d'enseignement dans des dizaines de classes et en confrontant les pratiques d'enseignement dans le premier degré et dans le second degré. Plusieurs projets impliquant des didacticiens du français, voire portés par des didacticiens du français, ont été soutenus et financés par l'Agence nationale de la recherche (ANR). Outre une recherche pionnière sur les littéracies universitaires, pilotée par I. Delcambre ${ }^{4}$, on peut citer plus récemment le projet Écriture scolaire et universitaire: Corpus, Analyses linguistiques, Modélisations didactiques (É-Calm) coordonné par C. Doquet, associant les équipes de Grenoble, Toulouse, Université Sorbonne Nouvelle - Paris 3 et Université Paris 8 Vincennes - Saint-Denis, qui a constitué et analysé de grands corpus d'écrits scolaires et universitaires. Du côté de la littérature, plusieurs recherches d'envergure ont également été initiées ces dernières années. Le projet Pratiques effectives de la lecture analytique dans le second degré en France et en Belgique (Pelas), initié par S. Ahr et qui implique une quinzaine de chercheurs de France et de Belgique, se penche sur les pratiques d'enseignement de la lecture analytique des textes littéraires ${ }^{5}$. Le projet Gary, quant à lui, réunit des chercheurs de Belgique, France, Québec, Suisse et interroge les compétences de lecture des élèves et les pratiques d'enseignement du littéraire, dans une centaine de classes ${ }^{6}$. À Montpellier, c'est aux pratiques d'enseignants du cycle 3 (dernières années de l'école primaire et première année du collège) que s'intéresse le projet $d u$ Texte à la Classe (Talc), dirigé par B. Louichon ${ }^{7}$. Quant au réseau Histoire de l'Enseignement des Littératures en Europe (Helice), impulsé en 2010 par M.-F. Bishop, il réunit des chercheurs européens de différents pays et vise une étude comparatiste de l'histoire de l'enseignement de la littérature à l'échelle européenne, à propos de différents objets disciplinaires (la fable, la lettre, l'extrait) ${ }^{8}$.

7 Enfin, du point de vue de la didactique du français comme discipline de formation, son institutionnalisation se poursuit malgré la multiplicité des réformes de la formation des enseignants en France. En Suisse avec les Hautes Écoles Pédagogiques (introduites au début des années 2000) ainsi qu'en Belgique, cette institutionnalisation va dans le sens d'une plus grande articulation entre recherche et formation notamment. En effet, la formation initiale et continue des enseignants est à la fois un lieu de diffusion des résultats des recherches en didactique du français langue première et un espace stratégique permettant aux chercheurs d'explorer la nécessité de nouvelles problématiques de recherche.

\section{Des numéros rétrospectifs pour combler l'amnésie}

$8 \mathrm{Au}$ cours de la dernière décennie, la didactique du français a ainsi poursuivi son processus d'institutionnalisation et cette discipline jeune, à laquelle B. Daunay et Y. Reuter (2011) reprochaient de ne pas suffisamment prendre en compte son histoire, a notamment comblé cette amnésie par la publication de numéros rétrospectifs dans deux revues importantes de didactique du français, Repères et Pratiques. Ces numéros bilans permettent à la fois de construire et de rappeler l'histoire de la didactique du français comme discipline de recherche et d'en désigner les zones éclairées ainsi que les impensés et zones d'ombre.

9 En 2012, le numéro 46 de la revue Repères intitulé "Vingt ans de recherche en didactique du français (1990-2010)» se propose de «centrer l'analyse sur l'évolution 
des objets didactiques et de leurs formes de traitement dans les recherches publiées » (Daunay \& Grossmann, 2012, § 11). Il comporte des notes de synthèse sur les recherches didactiques en grammaire (M.-L. Elalouf), sur la didactique du lexique (É. Nonnon), de l'écriture (C. Doquet) et de l'oral (R. Hassan) qui prennent comme corpus les publications dans cette revue depuis les années 1990. D'autres articles proposent un historique de l'évolution des recherches en didactique du français sur l'enseignement continué de la lecture dans l'espace romand (S. Aeby Daghé), la place accordée à l'école maternelle dans les publications de Repères (V. Boiron et B. Kervyn), les approches de la formation des enseignants en didactique du français (R. Gagnon et K. Balslev) ou les articles de didactique du français dans les sujets de concours de recrutement de professeurs des écoles en France entre 1994 et 2009 (F. Quet). Certaines contributions s'interrogent aussi sur des points aveugles. Ainsi M.-M. Bertucci et V. Castellotti questionnent la faible place accordée à la variation dans les recherches en didactique $\mathrm{du}$ français à travers l'analyse de trois revues de didactique du français langue première, Pratiques, Le français aujourd'hui et Repères. Les coordinateurs de ce numéro de Repères soulignent cependant le caractère partiel de cet état des lieux, qui s'appuie sur les réponses à l'appel à contribution et notent l'absence de champs importants dans la discipline comme l'orthographe ou la littérature par exemple, d'où le sous-titre choisi pour le numéro : «Quelques aspects des recherches dans les revues ».

De même, en avril 2015, la revue Pratiques a organisé un colloque pour célébrer les 40 ans de son existence: "Pratiques et l'enseignement du français : bilan et perspectives ", colloque qui a donné lieu à deux publications. Un numéro de la revue Pratiques regroupe les communications dans les domaines de l'étude de la langue, des textes et des discours. Un ouvrage collectif (Petitjean, 2016) regroupe les communications portant globalement sur la discipline « français » et sa didactique. Le titre de l'ouvrage, Didactiques du français et de la littérature, peut intriguer le lecteur dans la mesure où le pluriel semble acter l'autonomie de la didactique de la littérature par rapport à la didactique du français. De fait, si la deuxième partie de l'ouvrage fait un bilan des travaux en didactique de la littérature en tant que discipline de recherche, la première partie est plutôt centrée sur les articulations entre la discipline scolaire Français et la didactique du français comme discipline de recherche et de formation. Sont questionnées les évolutions des programmes ainsi que celles du recrutement et de la formation initiale des enseignants, y compris sous la plume de responsables institutionnels. Le numéro 169-170 de Pratiques (Masseron, 2016), qui est la seconde publication du colloque, est également organisé en deux grandes parties. La première partie regroupe des communications qui portent un regard rétrospectif sur les années d'émergence de la didactique $d u$ français langue première comme discipline de recherche, notamment sur la place de la grammaire textuelle, avec les contributions de J.-M. Adam, L. Lundquist, B. Combettes et J.-P. Bronckart. Des bilans sont proposés sur la didactique de l'oral (É. Nonnon), l'enseignement de la lecture (L. Sprenger-Charolles), de l'écriture (C. Garcia-Debanc) ou de l'orthographe (M. Fayol et J. Jaffré). La seconde partie du numéro propose des résultats de recherches actuelles dans les différents sous-domaines de l'étude de la langue, qui témoignent de la dynamique dans ce secteur de la didactique du français : grammaire (ateliers pour l'enseignement de la réflexion métalinguistique à l'école, enseignement du verbe, compléments du verbe, expansions du nom), orthographe (homophones grammaticaux), lexique (verbes de déplacement ou étymologie). M.-L. Élalouf réplique le dispositif proposé par J.-F. Halté (1982) pour comparer les annotations des enseignants sur les copies des élèves en 1982 et 
aujourd'hui. D'autres contributions exposent les résultats de recherches sur l'apprentissage du langage oral à l'école maternelle ou présentent les résultats de la recherche Ifé sur l'enseignement de la lecture-écriture au cours préparatoire.

\section{Le temps des hommages, des bilans et des dictionnaires}

11 Ces dernières années ont également été marquées par un phénomène qui, en lui-même, témoigne d'une forme d'institutionnalisation des recherches en didactique du français, à savoir les numéros de revues ou livres collectifs d'hommage à des acteurs importants du champ, à l'occasion de leur départ à la retraite. Déjà en 2006, la revue belge du Cedocef de Namur, Enjeux (Vrydaghs, 2006), avait dédié son numéro 66 à J.-M. Rosier, dont de nombreux contributeurs mettaient en avant l'engagement (Reuter, Petitjean, Dufays, Laurent, etc.), voire l'ethos militant (Daunay), ainsi que la diversité des domaines de recherche, entre littérature et didactique. La dernière décennie a vu se multiplier ces entreprises: Littérature, linguistique et didactique du français (Masseron, Privat \& Reuter, 2015) autour d'A. Petitjean, Théories-didactiques de la lecture et de l'écriture (Dias-Chiaruttini \& Cohen-Azria, 2017) autour d'Y. Reuter et Didactique du français et construction d'une discipline scientifique (Aeby Daghé et al., 2019) autour de B. Schneuwly. Les sommaires et la diversité des contributeurs témoignent - à l'instar du numéro en hommage à J.-M. Rosier - de l'intrication des domaines de recherche des trois chercheurs concernés, entre linguistique, littérature et didactique (voire psychopédagogie pour B. Schneuwly, dont c'est l'ancrage disciplinaire initial), et des relations tissées par la didactique du français avec ce qu'Y. Reuter (2007a) nomme les " disciplines contributoires ", et qui ont de fait participé de la construction épistémique et conceptuelle du champ de la didactique du français. Le statut d'" hommage » de ces ouvrages les oriente davantage vers un mélange de contributions sur des objets très divers que vers des ouvrages de synthèse, mais la récapitulation des parcours biographiques et scientifiques - passage obligé du genre - voire les entretiens avec les chercheurs destinataires de l'hommage, font de ces livres, des formes de «bilans d'étape " pour la discipline, comme l'écrivent A. Dias Chiaruttini et C. Cohen-Azria (2017, p. 10) dans leur introduction. Ainsi se dessine l'image complexe d'une discipline pour laquelle une " pluri- ou interdisciplinarité est nécessaire et possible » et " qui à la fois reconnait l'autonomie de la "didactique du français" tout en la combinant avec d'autres disciplines ", comme l'écrit B. Schneuwly $(2015$, p. 233) dans le texte qui clôt le volume autour d'A. Petitjean. L'intrication de la didactique $d u$ français avec les recherches littéraires ou linguistiques est en effet très nette dans cet ouvrage, dans la logique des travaux d'A. Petitjean lui-même et plus largement des choix scientifiques de Pratiques. Dans les deux autres (Dias-Chiaruttini \& Cohen-Azria, 2017 ; Aeby Daghé et al., 2019), le dialogue s'opère plutôt avec des didacticiens d'autres disciplines ou avec des chercheurs non francophones et cette diversité, qui correspond à la diversité des ancrages de ces chercheurs, témoigne également de cette interdisciplinarité constitutive du champ. Dans tous les cas, la construction de la didactique du français langue première comme discipline scientifique se fait par des dialogues théoriques et conceptuels avec des disciplines " contributoires " ${ }^{9}$ ainsi qu'avec d'autres didactiques ${ }^{10}$, y compris en dehors de l'espace francophone.

12 Parallèlement à ces livres d'hommage, certaines figures historiques du champ ont cherché à mettre à disposition des chercheurs une forme de synthèse de leurs travaux. Ainsi J.-P. Bronckart (2016) a-t-il rassemblé, sous un titre programmatique et malicieux 
(Pourquoi et comment devenir didacticien?), certains de ses textes « ayant trait au statut et aux orientations de la didactique»(ibid., p. 9), qu'il a fait précéder d'une riche introduction présentant son parcours scientifique de vygotskien devenu didacticien. De même, dans un ouvrage paru en 2016 intitulé De la pédagogie du français à la didactique des langues avec comme sous-titre "Les disciplines, la linguistique et l'histoire », J.L. Chiss a mis en perspective ses publications à différents moments de sa carrière pour montrer l'émergence de la didactique du français langue première et ses articulations avec la didactique du français langue étrangère et avec l'histoire des idées linguistiques.

De son côté, l'AIRDF a pris sa part dans cette volonté de rendre plus visibles et de capitaliser les travaux des chercheurs en didactique du français en organisant, par exemple en 2011, une journée d'études intitulée «La didactique du français à la croisée des chemins. Bilan et perspectives" afin de commémorer ses 30 ans d'activité scientifique et ses 25 ans de vie associative ${ }^{11}$; ou surtout en lançant à la fin des années 2000 une collection d'ouvrages de synthèse - à parution annuelle - sur des thématiques et des problématiques variées. Ainsi douze ouvrages ont-ils été publiés à ce jour : ils visent à alimenter non seulement des domaines ou des objets centraux de la didactique du français (l'oral, l'écriture de fiction, l'étude de la langue, les genres, l'évaluation, la littérature, etc.) mais aussi à interroger le champ même de la didactique du français, sa construction et ses frontières (à travers des ouvrages sur les concepts et méthodes ou sur les contextes et les réformes) ou ses relations avec d'autres disciplines ${ }^{12}$.

14 Enfin, cette volonté de proposer des travaux de synthèse est particulièrement perceptible dans les deux dictionnaires de didactique parus dans la période, même si leurs modalités de réalisation et leurs visées diffèrent sensiblement.

Le Dictionnaire des concepts fondamentaux des didactiques, édité par Y. Reuter en 2007 et rédigé par une équipe restreinte d'auteurs issus d'un même laboratoire de recherche, se propose de contribuer au projet comparatiste des didactiques. Bien qu'il soit à l'initiative d'un didacticien du français et que trois didacticiens du français s'inscrivent parmi les cinq auteurs (Y. Reuter, B. Daunay et I. Delcambre), ce Dictionnaire prend en effet en compte les didactiques des différentes disciplines et pas seulement la didactique du français. Il veut mettre à la disposition d'un public assez large (étudiants, formateurs, chercheurs, etc.) un état des travaux conduits dans le champ des didactiques des disciplines scolaires. Le pluriel même du titre, des didactiques, situe la didactique du français au sein des didactiques des différentes disciplines scolaires, et inscrit l'ouvrage dans le champ de la didactique comparée. En effet, sur les 40 concepts définis, peu émanent plus particulièrement de la didactique du français: "communauté discursive», «conscience disciplinaire», "genres du discours», "métalangage - activité métalinguistique » et "pratiques langagières ».. Le projet exposé dans la présentation est de présenter l'«armature conceptuelle fondamentale des didactiques » au moment de sa publication (Reuter, 2007a, p. 5).

Un dictionnaire de didactique de la littérature publié en 2019, grande entreprise collective coordonnée par N. Brillant-Rannou, F. Le Goff, M.-J. Fourtanier et J.-F Massol, est, quant à lui, le produit de contributeurs très nombreux et venus d'horizons très divers et s'attache à légitimer son champ de recherche. Il propose en 392 pages, des notices sur l'histoire de la didactique de la littérature dans son émergence, des jalons historiques et théoriques sur cette discipline avec, notamment, des regards croisés sur 
compréhension/interprétation et un ensemble de près de 80 notices critiques avec des entrées aussi diverses que "genres littéraires ", "numérique ", "objets sémiotiques secondaires (OSS)», "personnage», "stéréotype», "activité fictionnalisante du lecteur ", «empathie ", " écriture de la réception ", "non lecteur ", "spectature", « cercle et comité de lecture »... Une telle entreprise témoigne de la nécessité de faire un point de situation sur des concepts importants dans le champ de cette très jeune discipline qu'est la didactique de la littérature, mais aussi de la dynamique de ce champ, par le nombre de contributeurs et la grande pluralité des concepts et des points de vue qu'ils permettent sur la discipline.

\section{Les concepts en didactique du français, discipline de recherche}

La question des concepts, de leur définition, de leur réélaboration, mais aussi de leurs usages, est une question ancienne chez les didacticiens, qui accompagne la construction du champ de la didactique du français. Dès 1969, dans le premier numéro de Repères alors bulletin de liaison des équipes de l'INRP (Institut national de recherche pédagogique) -, H. Romian (1969, p. 3) se réjouit d'« un travail fondé sur des bases de plus en plus scientifiques ». Dans les revues qui apparaissent dans les décennies 1970-1980 (Le Français aujourd'hui en 1968, Repères en 1969, Pratiques en 1974, Enjeux en 1982, Recherches en 1984, La Lettre de la DFLM à la fin des années $1980{ }^{13}$ ), les articles théoriques et les notes de lecture ont toujours côtoyé les articles professionnels rendant compte de démarches et d'expérimentations en classe. Des comptes rendus de thèse ou d'habilitations à diriger des recherches soutenues en didactique du français qui, du fait de leur statut académique, explicitent tout particulièrement le cadre théorique et les concepts mobilisés - sont présents dans la Lettre de la DFLM dès le début des années 1990. La volonté de mettre en perspective et de faire apparaitre les cadres conceptuels et méthodologiques est particulièrement visible depuis une ou deux décennies comme nous l'avons montré supra. L'effort remarquable de nombreuses revues pour mettre leurs archives à disposition du plus grand nombre sur des plateformes ouvertes ${ }^{14}$ ou la création d'une section " archives » sur le site de l'AIRDF témoignent de ce même mouvement de capitalisation, qui participe de la légitimation du champ. Mais l'effort même de conceptualisation n'a pas attendu ces synthèses rétrospectives et contribue à l'émergence puis à la consolidation voire l'institutionnalisation de la didactique du français langue première depuis ses débuts.

\section{La circulation des concepts fondateurs entre les didactiques pionnières : les emprunts, les migrations et les concepts nomades}

Comme le faisaient remarquer B. Daunay, Y. Reuter et B. Schneuwly (2011, p. 25), si la didactique $d u$ français emprunte souvent alors qu'elle est peu prêteuse, ce n'est de toute façon pas une «logique comptable qui doit présider à [la] réflexion » sur les concepts :

Peu importe le nombre de concepts dont la didactique du français pourrait s'attribuer la paternité ; ce qui compte est la manière dont elle peut organiser son interprétation conceptuelle ${ }^{15}$. 

concepts proposés à la réflexion aux participants de la table ronde ("discipline», "progression » et "geste didactique ») ne sont pas non plus des concepts propres à la didactique du français. S'ils ont donné lieu à des travaux importants en didactique du français (par exemple pour le concept de « discipline » voir Falardeau et al., 2007 ; pour la « progression » voir Coste \& Véronique, 2000 et Nonnon \& Dolz, 2010 ; pour la notion de « geste " voir Bucheton \& Soulé, 2009 ; voir aussi Dupuy \& Soulé dans ce numéro), ces concepts ne sont pas spécifiques au champ, pas plus que le "rapport à ", que retravaille ici H. Le Levier. En réalité, la question n'est pas tant de savoir quels concepts sont inventés au sein de la didactique du français que de comprendre comment la didactique du français redéfinit, voire reconstruit, les concepts qu'elle utilise (voir dans ce numéro la contribution de B. Schneuwly et C. Ronveaux) et comment elle conceptualise certaines notions ou certains « concepts quotidiens » (c'est notamment le cas de la «discipline », dont C. Margolinas rappelle dans sa contribution que ce n'est pas un hasard si elle est questionnée par les didacticiens du français et très peu par les didacticiens des mathématiques). En réalité, une grande partie du travail conceptuel dans les didactiques disciplinaires relève d'emprunts, de reconstructions, de migrations, de ce "nomadisme " que mettait déjà en évidence I. Stengers (1987) et qui constitue l'un des ressorts du travail scientifique, tant que l'on se garde de toute naturalisation. De ce point de vue, les travaux comparatistes, qu'il s'agisse d'ouvrages ou de revues mettant en dialogue les différentes didactiques ou de colloques et journées d'études permettant des échanges entre didacticiens de différentes disciplines (voir les manifestations organisées par l'ARCD, par exemple), nourrissent et enrichissent le travail conceptuel commun ainsi que celui de chacune des didactiques.

ant, si la didactique du français n'a forgé, en tant que discipline de recherche, qu'un petit nombre de concepts nouveaux, comme par exemple ceux de « configuration didactique " (Halté, 1992) ou de "conscience disciplinaire» (Reuter, 2007b), elle a souvent reconfiguré des concepts venus d'ailleurs, qu'il s'agisse de concepts issus de la sociologie («rapport à»: voir dans ce numéro Le Levier) ou de la didactique professionnelle ("geste professionnel »). Ces reconfigurations ne sont pas de simples adaptations de concepts, mais des élaborations conceptuelles qui, d'ailleurs, peuvent à leur tour rendre poreuses les frontières entre les champs disciplinaires: comme le rappellent $\mathrm{C}$. Dupuy et $\mathrm{Y}$. Soulé dans ce numéro, les travaux sur les « gestes » venus de la didactique du français ont eu des répercussions bien au-delà, à la fois dans le champ de la recherche et dans celui de la formation.

De plus, la didactique du français interroge, depuis ses fondements, les limites de concepts issus d'autres didactiques disciplinaires tels que ceux de "transposition didactique » ou de "pratiques sociales de référence ", qui ont fait l'objet, depuis les années 1990, de travaux voire de réélaborations (voir dans ce numéro la contribution de N. Denizot). Le concept de "transposition didactique " est, de ce point de vue, particulièrement intéressant, à la fois par les discussions et débats qu'il a suscités au sein même de la communauté des didacticiens du français (voir notamment le numéro 97/98 de Pratiques entièrement consacré au sujet: Petitjean, 1998) mais aussi par les réélaborations et les propositions de concepts alternatifs qu'il a suscitées, du «traitement didactique " proposé par H. Romian dès les années 1980 (Romian, 1987) à l'« élaboration didactique » de J.-F. Halté (1992), en passant par les deux concepts de « recomposition et reconfiguration » promus par C. Vargas $(2014)^{16}$ et les réflexions sur 
les "pratiques sociales de référence» (Martinand, 1986). Deux articles du présent numéro (Schneuwly \& Ronveaux ; Denizot) montrent d'ailleurs que l'histoire n'est pas terminée, et que le travail d'élaboration conceptuelle ne cesse de se poursuivre.

\section{Concepts, méthodologies et données empiriques} sciences F. Pellaud, R.-E. Eastes et A. Giordan (s. d.) définissent différents «modèles de l'apprendre » comme des " outils", " une "aide à penser" pour mieux comprendre la réalité et, le cas échéant, intervenir sur elle ». En effet, un modèle scientifique a une fonction descriptive (il doit permettre de rendre compte de la réalité observée), explicative (il doit aider à la comprendre) et prédictive (il doit aider à prévoir les effets de la mise en place de tel ou tel paramètre). M. Thouin (2017, p. 109) précise qu'un modèle "est jugé surtout en fonction de la qualité des questions qu'il suscite, ou auxquelles il permet de répondre ». Ainsi dans sa définition scientifique le modèle est-il un système explicatif qui permet de rendre compte de certains phénomènes observés. B. Schneuwly et C. Ronveaux montrent bien dans ce numéro comment l'architecture conceptuelle permettant de rendre compte de la sémiotisation en jeu dans les activités d'enseignement s'est enrichie au fil des travaux de leur équipe de recherche pour devenir une "triple sémiotisation ", dont S. Aeby présente aussi tout l'intérêt pour analyser des pratiques de lecture littéraire au secondaire. De la même manière, la théorisation des gestes professionnels didactiques développée par les équipes de Montpellier (autour de D. Bucheton, Y. Soulé et C. Dupuy) et de Bordeaux (autour de J.P. Bernié, M. Jaubert et M. Rebière) s'est affinée grâce aux recherches empiriques, comme en porte trace la dénomination même de ce concept : du " geste professionnel » au «geste professionnel langagier didactique». Les modèles englobant plusieurs concepts sont proposés pour être opératoires dans une double perspective: la production de nouveaux résultats scientifiques et l'utilisation en analyse de pratiques dans les formations professionnelles au métier d'enseignant.

En revanche, d'autres concepts présents dans les contributions de ce numéro, comme celui de "problématisation» développé par M.-C. Pollet pour l'enseignement de l'écriture de recherche à des étudiants, sont beaucoup moins englobants. Ils sont plutôt utiles à la didactique en tant que discipline de formation pour rendre plus visibles des contenus de formation. 

même statut dans la discipline scientifique didactique du français, ni dans son état actuel et ni dans sa genèse. Il est pourtant d'une grande importance pour la didactique du français - et pour les didactiques en général, qui sont finalement des champs de recherche assez récents, mais qui arrivent, peut-être, à maturité - de prendre la mesure du chemin parcouru et non seulement de capitaliser les recherches, pour élaborer un cadre théorique qui leur soit propre, mais aussi de mettre en débat ce cadre théorique, pour éviter de naturaliser les notions et les concepts. Le présent numéro de Pratiques s'inscrit dans cette perspective, qui s'appuie sur l'histoire du champ : il nous a en effet paru important de prendre également en compte l'histoire de la constitution de notre discipline, et le lecteur pourra noter que de nombreuses contributions de ce numéro comportent un volet historique, notamment celles de M.-C.Guernier, N. Denizot, B. Schneuwly et C. Ronveaux, C. Garcia-Debanc.

Enfin, le questionnement sur les concepts ne peut être dissocié d'une réflexion épistémologique sur les méthodologies de la recherche en didactique du français langue première. Celles-ci ont considérablement évolué depuis une vingtaine d'années, au profit d'une plus grande rigueur et d'un meilleur contrôle des données collectées et analysées ${ }^{17}$. Les contributions de ce numéro témoignent de leur variété : étude qualitative des conceptions des élèves (H. Le Levier), observation et analyse de pratiques enseignantes (S. Aeby, J. Crinon \& C. Viriot-Goeldel) ou traitement qualitatif et quantitatif de productions scolaires dans une étude longitudinale sur deux années scolaires (M. Beaumanoir-Secq).

\section{Architecture du numéro et résumés des différents articles}

\section{Première section : Inventions, emprunts, migrations}

La première section regroupe des articles qui interrogent cette question des concepts en didactique du français - interrogation qui peut prendre la forme d'une mise en perspective historique et épistémologique - et/ou qui rendent compte de nouvelles élaborations ou reconstructions conceptuelles.

M.-C. Guernier propose une synthèse sur l'élaboration épistémologique de la didactique $d u$ français. Elle revient tout d'abord, sous forme d'une brève revue diachronique, sur quelques-uns des principaux textes et ouvrages fondateurs qui ont accompagné depuis les années 1990 la construction de la didactique du français comme champ disciplinaire autonome, et montre que cette construction épistémologique est encore émergente, même si elle fait largement consensus. Elle s'intéresse ensuite à la manière dont les travaux des didacticiens convoquent et définissent le concept même de concept, et le définissent - ou non - par rapport à la notion ou à la distinction concept scientifique/concept quotidien empruntée à L. Vygotski, avant d'explorer la nature et les modalités de la mise en œuvre de la conceptualisation, en tant qu'activité intellectuelle, dans quelques recherches et/ou métarecherches récentes en didactique du français.

N. Denizot vise à construire le concept de "culture scolaire» (Denizot, 2021) en didactique du français - et plus largement dans le champ des didactiques disciplinaires -, à partir des élaborations conceptuelles proposées par les historiens des 
disciplines et les sociologues de l'éducation. Elle définit la culture scolaire comme un produit original de l'école, élaboré pour des finalités qui sont propres à cette dernière, et articule ainsi ce concept de culture scolaire à celui de "scolarisation", qui désigne les processus complexes de construction de la culture scolaire. Ce faisant, l'auteure propose un bref retour sur le concept de "transposition didactique», en tant que concept fondateur dans les champs des didactiques pour penser la nature et la construction des savoirs scolaires, ainsi que sur les débats qu'il a suscités en didactique du français.

31 Ce concept de "transposition didactique » est au cœur de l'article de B. Schneuwly et C. Ronveaux, qui revient sur le système conceptuel forgé par l'équipe de recherche genevoise, dont les travaux articulent le concept de transposition didactique avec une approche instrumentale empruntée à L. Vygotski - articulation qui est explicitée et discutée en conclusion. Les auteurs présentent ce système conceptuel en sept thèses, qui sont l'occasion à la fois de revenir sur les travaux menés au sein de l'équipe genevoise ces dernières décennies ainsi que de présenter et définir les concepts qui ont été élaborés ou reconstruits à l'occasion de ces recherches, comme l'objet et la séquence d'enseignement, l'archiélève, la sédimentation, la disciplinarisation et la disciplination.

Y. Vuillet et B. Védrines développent une analyse théorique à la fois compréhensive et critique de la rencontre de la didactique et de la littérature, sous l'angle des processus d'assujettissement par lesquels se constituent des sujets. Définissant la didactique comme une science sociohistorique caractérisée par de nombreux modèles complémentaires voire concurrents, ils visent à construire une théorie du sujet qui soit opératoire en didactique et qui permette de modéliser et de décrire des phénomènes didactiques liés à la "réputation littéraire » - concept qui permet de questionner et dénaturaliser le problème de la définition de "la» littérature. Ainsi élaborent-ils une théorie du « sujet didactique » qui prend en compte le système didactique et qui ne se cantonne pas au sujet apprenant mais qui s'étend au sujet enseignant.

Le dernier article de la section propose une retranscription d'une table ronde organisée par J.-L. Dufays lors du colloque. Les trois participants, O. Dezutter, M.-L. Élalouf et F. Le Goff, ont été invités à revenir sur trois concepts souvent mobilisés dans les travaux actuels en didactique du français, à savoir : la " discipline ", la "progression " et le "geste didactique ». Pour chacun de ces concepts, les auteurs en analysent tout d'abord l'importance, à leurs yeux, dans le champ de la didactique du français, puis explicitent l'approche qu'ils en ont ainsi que le cadre théorique dans lequel ils l'inscrivent, avant de le discuter ou de faire état des questions qu'il suscite pour eux.

\section{Deuxième section : Concepts et modèles}

C. Garcia-Debanc rappelle l'historique et la définition de trois concepts proposés par des didacticiens du français : «configuration didactique " (Halté, 1992), " conscience disciplinaire » (Reuter, 2007b) et "modèles disciplinaires en actes» (Garcia-Debanc, 2007 ; 2019). Elle montre comment ils rendent compte de trois niveaux de la transposition didactique (Chevallard, 1985-1991). Elle les met à l'épreuve de données collectées dans des classes de fin d'école primaire françaises auprès de professeurs des écoles stagiaires et de leurs élèves. L'analyse de ces données fait apparaitre les zones de flou et de tension dans la mise en œuvre de la discipline « français » à l'école primaire 
et conduit à la formulation de points de vigilance pour la formation initiale des enseignants du premier degré.

Les deux articles suivants, celui de C. Dupuy et $\mathbf{Y}$. Soulé et celui de M. Jaubert et M. Rebière exposent deux modèles élaborés en didactique du français dans les années 2000 respectivement par l'équipe de Montpellier autour de D. Bucheton et par celle de Bordeaux, autour de J.-P. Bernié, pour rendre compte de l'activité de l'enseignante dans la classe et des conditions didactiques favorables aux apprentissages des élèves.

C. Dupuy et Y. Soulé présentent le concept de "gestes professionnels », décliné plus tard en « gestes professionnels langagiers didactiques » et en rappellent la genèse. Ils mettent en évidence le projet initial des chercheurs, qui est d'analyser et de comprendre le métier enseignant dans sa complexité et ils explicitent les concepts connexes de "préoccupations " (avec le multi agenda de l'enseignant- présentant les cinq préoccupations enchevêtrées) et de "postures" comme manières d'être, de penser et d'agir (Bucheton, Dezutter, 2008; Bucheton 2019). Leur exposé met en évidence la solidarité entre le concept de geste professionnel et l'ensemble de l'appareil conceptuel dans lequel il s'inscrit, tout en montrant également les problèmes et les limites dans l'utilisation de ces concepts. Ils illustrent enfin la dimension opératoire de ce modèle, ce qu'ils appellent sa "rentabilité », par des extraits de travaux d'étudiants de master et de thèses. Ils insistent tout particulièrement sur la dimension opératoire de ce concept en formation initiale et continue d'enseignants pour formaliser et expliciter des gestes de métier et les transformer en gestes professionnels, avec la dimension réflexive que suppose leur maitrise.

En lien avec les travaux de l'équipe de Montpellier, avec laquelle ont été inscrites des collaborations dans le cadre d'une équipe de recherche en technologie pour l'éducation (Érté) dans les années 2000, M. Jaubert et M. Rebière présentent l'appareil conceptuel mis au point par l'équipe de Bordeaux pour montrer le rôle essentiel du langage dans la construction des connaissances scolaires dans les différents domaines disciplinaires, au croisement notamment des travaux de L. Vigotsky et de M. Brossard sur le langage, de J.-B. Grize sur l'énonciation et de J. Bruner sur l'étayage. Le concept central de "communauté discursive", emprunté à D. Maingueneau se spécifie en communauté discursive scolaire disciplinaire. Trois schématisations successives du modèle permettent de montrer comment s'articulent les différentes composantes et mettent en évidence le rôle pivot des gestes professionnels langagiers didactiques. La pertinence de ces concepts est illustrée par des exemples empruntés à l'enseignement du français (enseignement grammatical et écriture fictionnelle) et à l'enseignement des sciences aux cycles 2 et 3 de l'école primaire.

La contribution de $\mathbf{S}$. Aeby-Daghé s'inscrit également dans un modèle, celui de l'équipe de Genève autour de B. Schneuwly et J. Dolz et d'une théorie : celle de la double et même triple sémiotisation. Ici, le concept central est celui d'outil au sens vygotskien, le projet scientifique étant « la description des outils disciplinaires mis en œuvre dans les classes par les enseignantes de français ». Les données empiriques recueillies et analysées sont numériquement importantes: 61 périodes de 45 minutes dans des classes de l'enseignement secondaire obligatoire en Suisse avec des élèves de 15 à 16 ans. Elles sont constituées par des suites de leçons consacrées à la lecture et à l'étude de quatre textes contrastés (un conte philosophique, un roman policier, une autobiographie et un éditorial), dont 32 séances consacrées à la lecture du conte de 
Voltaire Candide ou l'optimisme dans trois classes. L'analyse de ce corpus a permis à la chercheuse d'inventorier 12 genres d'activité scolaire (GAS) dans l'activité de lecture littéraire au secondaire obligatoire, auxquels s'ajoutent six GAS correspondant à des approches plus innovantes. La chercheuse applique cette typologie à trois séquences sur Candide, procède à une analyse quantitative et catégorise les GAS recensés en trois types de sémiotisation : (a) des activités de présentification visant à l'appropriation du texte, (b) des activités de pointage liées au commentaire et (c) un discours visant à assurer l'ancrage énonciatif et le sens des activités. L'article se termine par une analyse qualitative de la transcription d'un débat interprétatif dans l'une des classes, à propos de la chute de Candide: « il faut cultiver son jardin ».

\section{Troisième section : Des concepts pour dialoguer avec d'autres disciplines}

39 La troisième section du numéro regroupe trois articles qui mettent à l'épreuve le nomadisme ou la migration des concepts, en envisageant la manière dont la didactique du français s'enrichit de cadres théoriques et conceptuels venus d'ailleurs, qu'il s'agisse de la didactique des mathématiques, de la didactique du FLES (français langue étrangère et seconde) ou du champ des littéracies.

c. Margolinas, didacticienne des mathématiques, présente dans son article des concepts issus de la théorie des situations et diffusés au-delà de la didactique des mathématiques, pour questionner leur transposition dans le champ de la didactique du français, à partir notamment de travaux qu'elle a menés en collaboration avec une didacticienne $\mathrm{du}$ français, M. Laparra. Elle montre comment la distinction fondamentale faite par G. Brousseau entre "savoir» et "connaissance " permet d'éclairer la complexité des savoirs dans la classe de français, entre des savoirs assujettis, à l'université, à des institutions diverses, langue et littérature, et des connaissances situationnelles, dont elle discute ensuite le caractère "disciplinaire ». Prenant tout d'abord l'exemple d'une tâche courante en petite section, écrire son prénom avec des étiquettes-lettres, elle analyse les connaissances en jeu, qui ne relèvent pas toutes de la discipline « français ». Un deuxième exemple, la segmentation de mots à partir d'un modèle en grande section, permet de questionner l'adéquation des connaissances que doivent investir les élèves avec les savoirs en jeu pour l'enseignant.

41 Partant du constat que les recherches en littéracie sont en pleine expansion depuis plusieurs décennies, P. Dupont s'intéresse à la migration du concept de littératie et du champ des recherches dans ce domaine vers la didactique du français. Après un bref retour définitionnel sur la notion même de «littératie ", il explore, dans un second temps, à travers un corpus de revues, l'installation de la littératie dans le paysage des recherches en didactique du français puis la manière dont les didacticiens se sont emparés de ce champ conceptuel et les usages qui en sont faits. Enfin, dans une partie plus prospective, il cherche à montrer les apports euristiques de l'espace conceptuel de la littératie pour la didactique du français : selon l'auteur, en favorisant une approche intégrative de la discipline, la littératie ouvrirait de nouveaux lieux d'intervention didactique.

M.-A. Camussi-Ni plaide pour une meilleure communication entre didactique du français langue première et didactique du français langue étrangère. Elle montre que 
certains concepts fréquemment utilisés dans l'enseignement des langues étrangères, comme celui de fossilisation, pourraient avantageusement éclairer des problèmes d'apprentissage rencontrés en langue première. Elle montre, à l'aide d'exemples d'exercices proposés dans des manuels d'école primaire ou de lycée, que dans de nombreux cas les réponses attendues reposent sur une intuition linguistique que n'ont pas certains élèves de milieux populaires et qui n'ont pas fait l'objet d'un enseignement. Elle interroge la didactique $d u$ français langue première sur une prise en compte insuffisante de la dimension sociolinguistique.

\section{Quatrième section : Des concepts pour penser les situations d'enseignement}

La contribution de $\mathbf{J}$. Crinon et $\mathbf{C}$. Viriot-Goeldel fait apparaitre l'intérêt et les limites d'un dispositif. Elle prend appui sur les données collectées dans le cadre du projet Twictée soutenu par un financement de l'ANR, qui propose des échanges électroniques relatifs aux justifications métagraphiques des choix orthographiques dans des dictées également conçues en collaboration avec l'aide des réseaux sociaux. Après avoir situé la genèse du concept de « dispositif » et en avoir interrogé la dimension épistémologique par contraste avec ceux d'« outil» et de "milieu», utilisés préférentiellement par d'autres didacticiens ${ }^{18}$, les auteurs mettent en évidence la variété des mises en œuvre de ce dispositif dans les classes observées et les effets différentiels de ces mises en œuvre sur les apprentissages des élèves, notamment auprès des populations sociologiquement défavorisées et scolairement fragiles. L'étude s'appuie sur l'analyse quantitative de l'évolution des performances orthographiques d'élèves de $\mathrm{CM}^{19}$ dans des dictées et sur l'analyse qualitative d'extraits d'entretiens avec des enseignants et des élèves ainsi que de transcriptions d'interactions dans les classes. L'étude conclut à une importance primordiale d'une appropriation par les enseignants des enjeux du dispositif et de ses soubassements théoriques, au-delà d'une magie de l'outil numérique et sur la grande variabilité des mises en œuvre, aux antipodes des injonctions anglosaxonne relatives aux dispositifs.

M. Beaumanoir-Secq analyse aussi les effets d'un dispositif didactique : dans le cadre d'une recherche doctorale, elle a procédé à un suivi longitudinal des élèves d'une classe située en zone d'éducation prioritaire, avec une même enseignante, sur une durée de deux années, en CE2 et CM1, au cours d'une activité grammaticale, le tri de mots, qui consiste à catégoriser les différents mots d'une phrase ou d'un court texte. L'analyse statistique de l'évolution des performances des élèves relatives à l'identification correcte de la catégorie grammaticale d'un mot lui permet de mettre en évidence les difficultés relatives d'identification des différentes classes grammaticales et de définir les éléments d'une progressivité dans leur acquisition. Cette approche quantitative s'accompagne d'une étude qualitative des classifications proposées par les élèves et de la nature des justifications formulées. Cet article montre tout l'intérêt d'une approche longitudinale sur une durée longue, ce qui est assez rare encore dans les recherches en didactique du français langue première et celui d'associer une étude quantitative à une approche qualitative.

M.-C. Pollet et C. Glorieux s'intéressent aux notions de problématique et de problématisation dans l'écriture de recherche, en tant que celles-ci sont à la fois incontournables et sources de difficultés pour les étudiants. Dans une première partie, 
elles explorent tout d'abord les représentations que les étudiants ont de ces notions, avant de discuter certains travaux sur la question et de revenir sur certaines des définitions. Elles proposent ensuite une caractérisation théorique de la «problématisation » à partir de notions qui lui sont liées : "cadrage ", « niche » et "positionnement». Enfin, une dernière partie s'attache à une didactisation de la notion de problématisation et analyse des exemples d'activités conçues à cet effet, en compréhension et en production d'écrit, articulées à la découverte de la pratique de la recherche dans des champs disciplinaires divers.

H. Le Levier reprend le concept de « rapport à " pour interroger les représentations sur l'orthographe et son enseignement d'élèves de fin de collège (troisième) et d'étudiants post-bac dans des classes de techniciens supérieurs. Dans une première partie de l'article, elle rappelle la fécondité de ce concept emprunté à la sociologie des pratiques culturelles en didactique du français langue première dans les travaux sur l'écriture, notamment ceux de C. Barré de Miniac. Elle croise les réponses obtenues à partir de questionnaires et d'entretiens pour rendre compte de leur rapport à l'orthographe et les interroge aussi sur leur rapport à la réforme proposant des rectifications orthographiques. Ces données lui permettent de questionner «la mise en œuvre la norme orthographique comme pratique sociale ».

B. Kervyn plaide pour l'avènement d'un nouveau concept, celui de «préparation à l'écriture » en lieu des concepts jusque-là utilisés en didactique du français, ceux de «brouillon » ou d'" avant-texte », empruntés à la critique génétique. Cette nouvelle conceptualisation marque l'autonomisation de la didactique du français comme discipline de recherche mais permet aussi de rendre compte de phénomènes très spécifiques à l'activité d'enseignement, en particulier dans les premiers apprentissages de la lecture écriture, et ainsi, selon l'auteure, de mettre la focale sur des points impensés avec l'ancienne conceptualisation. De plus, du point de vue de la didactique du français comme discipline de formation, ce concept semble à l'auteure présenter l'avantage d'une dénomination plus claire et plus opératoire pour les professionnels de l'enseignement.

o. Tremblay propose également un nouveau concept, celui de "sensibilité lexicale ", pour dénommer une des composantes de la compétence lexicale, celle de s'intéresser aux mots, aussi bien dans leur dimension morphologique que sémantique. La référence à ce concept permet à la fois de rendre compte de remarques d'enfants et de définir un enjeu original en formation continue d'enseignants d'école primaire. La chercheuse insère la sensibilité lexicale dans un modèle d'ensemble de la didactique du lexique dont elle inventorie les connaissances et les habiletés. Cette sensibilité lexicale se décline sous un ensemble d'attitudes qu'elle recense également. Dans une dernière section de l'article, elle s'interroge sur le statut de la «sensibilité lexicale » comme «concept didactique» et elle conclut sur sa pertinence pour rendre compte des dimensions affectives dans les apprentissages.

DENIZOT N., DUfAYS J.-L. \& LOUICHON B. (dir.) (2019). Approches didactiques de la littérature. Namur : Presses Universitaires de Namur 


\section{BIBLIOGRAPHIE}

AEBY DAGHÉ, s. et al. (dirs) (2019). Didactique du français et construction d'une discipline scientifique. Dialogues avec Bernard Schneuwly. Villeneuve d'Ascq : Presses Universitaires du Septentrion. AHR, S. (2015). Enseigner la littérature aujourd'hui : « disputes » françaises. Paris : H. Champion. BELHADJIN, A. \& PERRET, L. (2020). L'Extrait et la fabrique de la littérature scolaire, Bruxelles : P. Lang. BRILLANT RANNOU, N. et al. (dirs) (2019). Un dictionnaire de didactique de la littérature, Paris : H. Champion.

BRONCKART, J.-P. (2016). Pourquoi et comment devenir didacticien? Villeneuve d'Ascq : Presses Universitaires du Septentrion.

BUCHETON, D. (2019). Les Gestes professionnels dans la classe. Éthique et pratiques pour les temps qui viennent, Paris : ESF

BUCHETON, D. \& DEZUTTER, O. (2008). Le Développement des gestes professionnels dans l'enseignement du français. Un défi pour la recherche et la formation, Bruxelles : De Boeck.

BUCHETON, S. \& SOULÉ, Y. (2009). « Les gestes professionnels et le jeu des postures des enseignants dans la classe : un multi-agenda de préoccupations enchâssées ». Éducation \& didactique 3, p. 29. En ligne : https://www.cairn.info/revue-education-et-didactique-2009-3-page-29.htm.

CHEVALLARD, Y. (1991) [1985]. La Transposition didactique : du savoir savant au savoir enseigné. Grenoble : Éd. La Pensée sauvage.

CHISS, J.-L. (2016). De la pédagogie du français à la didactique des langues. Les disciplines, la linguistique et l'histoire. Paris : Éd. L’Harmattan.

COSTE, D. \& VÉRONIQUE, G. D. (2000) (dirs). La Notion de progression. Fontenay-aux-Roses : ENS éd. DAUNAY, B. (2007). «État des recherches en didactique de la littérature ». Revue française de pédagogie 159, p. 139-189. En ligne : https://doi.org/10.4000/rfp.1175.

DAUNAY, B. \& REUTER, Y. (2011). « De quelques obstacles rencontrés par les recherches en didactique du français ». Pratiques 149-150, p. 9-24. En ligne : https://doi.org/10.4000/pratiques. 1680.

DAUNAY, B., REUTER, Y. \& SCHNEUWLY, B. (dirs) (2011). Les Concepts et les méthodes en didactique du français, Namur : Presses universitaires de Namur.

DAUNAY, B. \& GROSSMANN, F. (2012) « Vingt ans de recherches en didactique du français (1990-2010) », Repères. Recherches en didactique du français langue maternelle 46, p. 7-16. En ligne : https://doi.org/10.4000/reperes.82.

DELCAMBRE, I \& LAHANIER-REUTER, D. (dirs) (2012). « Littéracies universitaires ». Pratiques 153-154, p. 3-19. En ligne : https://doi.org/10.4000/pratiques.1905.

DENIZOT, N. (2021). La culture scolaire : perspectives didactiques. Pessac : Presses universitaires de Bordeaux.

DEZUTTER, O. (2004). « La didactique du français, emprunteuse mais aussi prêteuse. De nombreux emprunts mais quel apport dans la formation générale des maitres ? ». In : C. Lessard (éd.), Entre sens commun et sciences humaines. Quels savoirs pour enseigner? Louvain-la-Neuve : De Boeck Supérieur, p. 201-220. 
DIAS-CHIARUTTINI, A. \& COHEN-AZRIA, C. (dirs) (2017). Théories-didactiques de la lecture et de l'écriture? Fondements d'un champ de recherche en cheminant avec Yves Reuter. Villeneuve d'Ascq : Presses universitaires du Septentrion

DUFAYS, J.-L. et al. (2020). «Quelle progression des compétences interprétatives des élèves de 12 à 15 ans et quelle prise en compte par leurs enseignants? ». Pratiques 187-188. En ligne : https:// doi.org/10.4000/pratiques.9007.

DUMORTIER, J.-L. (éd.) (2011), La Lettre de l'AIRDF 50. En ligne : https://www.persee.fr/issue/ airdf_1776-7784_2011_num_50_1.

FALARDEAU, E. et al. (dirs) (2007), La Didactique du français. Les voies actuelles de la recherche. SainteFoix : Presses de l’Université Laval.

GARCIA-DEBANC, C. (2007). « Les modèles disciplinaires en acte dans les pratiques effectives d'enseignants débutants ». In : É. Falardeau et al. (dirs) La Didactique du français. Les voies actuelles de la recherche. Laval : Presses de l'Université Laval, p. 42-61.

GARCIA-DEBANC C. (2019). «Le français tel qu'on l'enseigne : objets enseignés en classe de français et modèles disciplinaires ». In : S. Aeby-Daghé, E. B. Bronckart, G. S. Cordeiro, J. Dolz et alii (dirs). Didactique du français et construction d'une discipline scientifique. Dialogues avec Bernard Schneuwly. Villeneuve d'Ascq : Presses Universitaires du Septentrion, p. 181-196.

GARCIA-DEBANC, C. \& MASSERON, C. (dirs) (2008). « La didactique du français ». Pratiques 1387-138. En ligne : https://doi.org/10.4000/pratiques.1147.

GoIGOUX, R. (2015). Lire et écrire. Rapport de recherche. Lyon : Ifé. En ligne : http://ife.ens-lyon.fr/ife/ recherche/lire-ecrire.

halté, A. \& PETITJEAN, A. (dirs) (2010). « Didactique du français (1) ». Pratiques 145-146. En ligne : https://doi.org/10.4000/pratiques.1481

halté, A. \& Petitjean, A. (dirs) (2011). « Didactique du français (2) ». Pratiques 149-150. En ligne : https://doi.org/10.4000/pratiques.1677

HALTÉ, J.-F. (1982) « Travailler en projet ». Pratiques 36, p. 38-77. En ligne : https://www.persee.fr/ issue/prati_0338-2389_1982_num_36_1.

HALTÉ, J.-F. (1992). La Didactique du français. Paris : PUF.

LE GOFF, F. et al. (dirs) (2020). «Enseignement du texte littéraire dans l'espace francophone : pratiques, formation, recherche ». Pratiques 187-188. En ligne $:$ https://doi.org/10.4000/pratiques. 8757

Louichon, B. (dir.) (2020). Un texte dans la classe. Pratiques d'enseignement de la littérature au cycle 3 en France. Bruxelles : P. Lang.

MARTINAND, J.-L. (1986). Connaitre et transformer la matière. Berne : P. Lang.

MASSERON, C. (dir.) (2016), « Enseignement/apprentissage de la langue, des textes et des discours. 40 ans de Pratiques ", Pratiques 169-170. En ligne : https://doi.org/10.4000/pratiques.2925

MASSERON, C., PRIVAT, J.-M. \& REUTER, Y. (dirs) (2015). Littérature, linguistique et didactique du français. Les travaux Pratiques d'André Petitjean. Villeneuve d'Ascq : Presses Universitaires du Septentrion. NONNON, É. (2010). «Quelle articulation entre la formation des maitres et la recherche en didactique du français ? », Pratiques 145-146, p. 38-44. En ligne : https://journals.openedition.org/ pratiques/1498 
NONNON, É. \& DOLZ, J. (2010) (dirs). « La notion de progression dans la pratique et la réflexion sur la langue de l'école au collège ». Repères. Recherches en didactique du français langue maternelle 41 . En ligne : https://doi.org/10.4000/reperes.273.

PELLAUD, F., EASTES, R.-E. \& GIORDAN, A. (s. d.). « Des modèles pour comprendre l'apprendre : de l'empirisme au modèle allostérique ». En ligne : https://www.andregiordan.com/articles/ apprendre/modalost.html

PETITJEAN, A. (dir.). (2016). Didactiques du français et de la littérature. Metz : Université de Lorraine.

REUTER Y. (dir.) (2007a). Dictionnaire des concepts fondamentaux des didactiques. Bruxelles : De Boeck.

REUTER, Y. (2007b). « La conscience disciplinaire », Éducation \& didactique, 1-2, p. 55-71. En ligne :

https://doi.org/10.4000/educationdidactique.175.

ROMIAN, H. (1969). « Un bulletin pourquoi pas ? ». Repères. Recherches en didactique du français langue maternelle 1, p. 2-3. En ligne : https://doi.org/10.3406/reper.1969.958

ROMIAN, H. (1987). « Aux sources des savoirs à enseigner : traditions scolaires, pratiques sociales, référents théoriques ». Repères. Recherches en didactique du français langue maternelle 71, p. 91-102. En ligne : www.persee.fr/doc/reper_0755-7817_1987_num_71_1_1904

RONVEAUX, C. \& SCHNEUWLY, B. (dirs) (2018). Lire des textes réputés littéraires : disciplination et sédimentation. Enquête au fil des degrés scolaires en Suisse Romande. Bruxelles : P. Lang.

SCHNEUWLY, B. (2015). « "La didactique du français comme champ de recherche autonome et spécifique” (Petitjean, 1985) : une utopie devenue (partiellement) réalité ». In : Masseron, C., Privat, J.-M. \& Reuter, Y. (dirs), Littérature, linguistique et didactique du français. Les travaux Pratiques d'André Petitjean. Villeneuve d'Ascq : Presses du Septentrion, p. 225-235.

SIMARD, c. et al. (2019). Didactique du français langue première. Bruxelles : De Boeck.

STENGERS, I. (dir.) (1987). D'une science à l'autre. Des concepts nomades. Paris : Éditions du Seuil.

THOUIN, M. (2017). «L'épistémologie et la recherche en didactique : les visées des sciences et les modèles ». Canadian Journal for New Scholars in Education 8 (1), p. 107-115. En ligne : https:// journalhosting.ucalgary.ca/index.php/cjnse/article/view/42174.

VARGAS, C. (2014). Les Grammaires scolaires. De la recomposition à la reconfiguration. Aix-en-Provence : Presses universitaires de Provence.

VRYDAGHS D. (2006), « Enseignement et engagement. Hommage à Jean-Maurice Rosier ». Enjeux. Revue de formation continuée et de didactique du français 66.

\section{NOTES}

1. Voir son site : https://airdf.ouvaton.org/

2. Le rapport (Goigoux, 2015) est consultable sur le site de l'Ifé : http://ife.ens-lyon.fr/ ife/recherche/lire-ecrire/rapport/rapport.

3. Voir Ronveaux et Schneuwly (2018).

4. Voir Delcambre \& Lahanier-Reuter (2012).

5. Un colloque en 2019 a donné lieu à un numéro de Pratiques (Le Goff et al., 2020).

6. Pour une présentation du projet et de premiers résultats, voir Dufays et al. (2020). 
7. Les résultats de cette recherche ont fait l'objet d'un ouvrage (Louichon, 2020).

8. Voir la dernière publication : Belhadjin \& Perret, 2020.

9. Voir, par exemple, l'article «Science didactique et réputation littéraire : le sujet en question » par $Y$. Vuillet et $B$. Védrines dans le présent numéro.

10. Voir par exemple la troisième section de ce numéro : « Des concepts pour dialoguer avec d'autres disciplines" ".

11. Voir le numéro 50 de La Lettre de l'AIRDF (Dumortier, 2011).

12. Voir la liste des ouvrages parus sur le site de l'AIRDF : https://airdf.ouvaton.org/ index.php/la-collection

13. Le premier numéro de la Lettre de la DFLM sur Persée est le numéro 6 datant de 1990.

14. Les archives de Pratiques, Repères et de la Lettre de l'AIRDF sont sur Persée. Celles de Recherches sont sur le site de la revue.

15. Ce sont eux qui soulignent.

16. Sur tout cela, voir Denizot, 2021.

17. Pour une synthèse sur les méthodologies les plus fréquemment utilisées en didactique du français langue première, on pourra consulter Simard et al., 2019, p. 383-392.

18. L'équipe genevoise autour de B. Schneuwly et J.Dolz s'appuie sur le concept d'« outil» dans un héritage vygoskien. Le didacticien des mathématiques, G. Brousseau a, de son côté, proposé une théorisation du «milieu » pour rendre compte de ce qu'il appelle les « situations adidactiques ».

19. Le «cours moyen » en France correspond aux deux dernières années de l'école primaire.

\section{AUTEURS}

\section{NATHALIE DENIZOT}

Sorbonne Université, Inspé de Paris, CELLF, F-75016 Paris, France

\section{CLAUDINE GARCIA-DEBANC}

Université de Toulouse, Inspé Toulouse Occitanie-Pyrénées, CLLE, F-31400 Toulouse, France 